\title{
ON THE PRIMARY IMPORTANCE OF PULSARS WITH FIVE-COMPONENT PROFILES
}

\author{
JoAnNa M. RANKIN \\ Department of Physics, University of Vermont
}

\begin{abstract}
Some 15 members of the class of profiles with five components have been identified. These profiles have a central core component and two pairs of conal outriders, thus exhibiting the highest degree of formal complexity observed. No good examples of pulsars with six or more profile components are known.

Members of the five-component class have very similar physical parameters and emission-region geometries. An analysis of their emission geometry indicates both that the core and conal beams scale as $P^{-1 / 2}$ and that the $1-\mathrm{GHz}$ conal emission comes from a height of some $100-200 \mathrm{~km}$.
\end{abstract}

\section{Introduction}

More than 15 pulsars have been identified which appear to be members of the five-component (M) profile species. These profiles all have a single, central core component and two pairs of conal outriders. Apparently, they result from a double-conal geometry as shown in figure 1 .

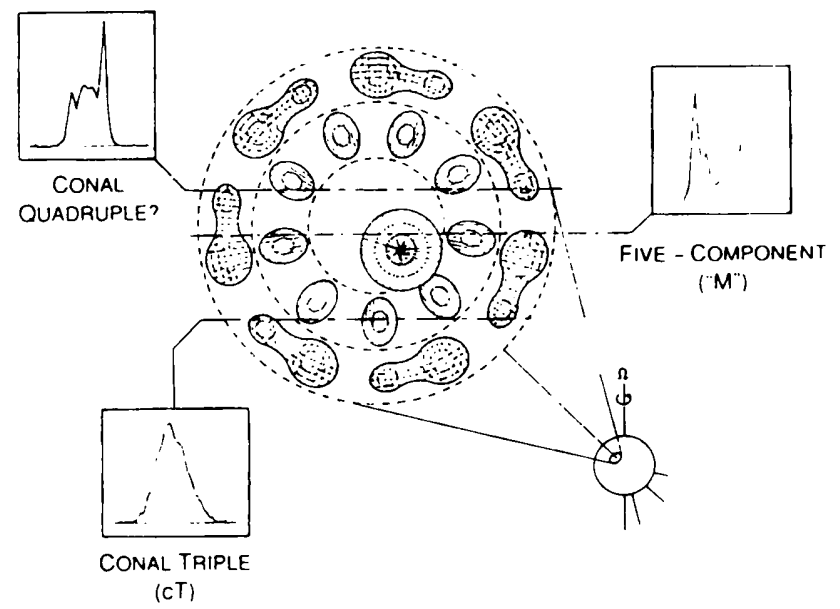

Figure 1 Schematic diagram showing the geometry of both the core beam and the double-conal beam. Central traverses of the sight line give a five-component profile; more oblique traverses provide other, entirely conal possibilities. Several examples of conal triple (cT) profiles have been identified, but no conal quadruple $(\mathbf{Q})$ stars have yet been positively identified.

M stars emit both core and conal radiation in roughly comparable amounts and exhibit a number of other similar physical and geometrical characteristics which are outlined below.

These five-component (M) pulsars seem to exhibit the highest degree of profile complexity observed. No class of pulsars - or even a single convincing example - with six or more components is known. No pulsars with four component (Q) pro- files have been positively identified, although several candidates are now known.

\section{Five-component profile prop- erties}

The five-component pulsars exhibit very similar profile characteristics as follows:

- two-pairs of conal components and one central core component-all roughly comparable (within factors of 2) in intensity. This is illustrated for pulsar $1237+25$ in figure 2 .

- as usual the core component tends to have a softer spectrum and thus becomes ever less prominent at high frequency and ever more dominant at low frequencies. (In PSR $1237+25$ this evolution is confused by profile mode changes; note the relative height of the core component in the 280 and 229$\mathrm{MHz}$ profiles in figure 2. Other profiles observed at about $100 \mathrm{MHz}$ have a much brighter core component.)

- at high frequencies the five components often merge into an overall "boxy" form.

- at low frequencies the inner conal components $(2$ and 4) sometimes become weaker faster than the outer pair ( 1 and 5 ).

- sense-reversing circular polarization associated with the central core component. See figures 3-5.

- a $\lesssim 180^{\circ}$, "S"-shaped traverse of the linear polarization angle, indicating that the sight line to the pulsar cuts the hollow conical emission 


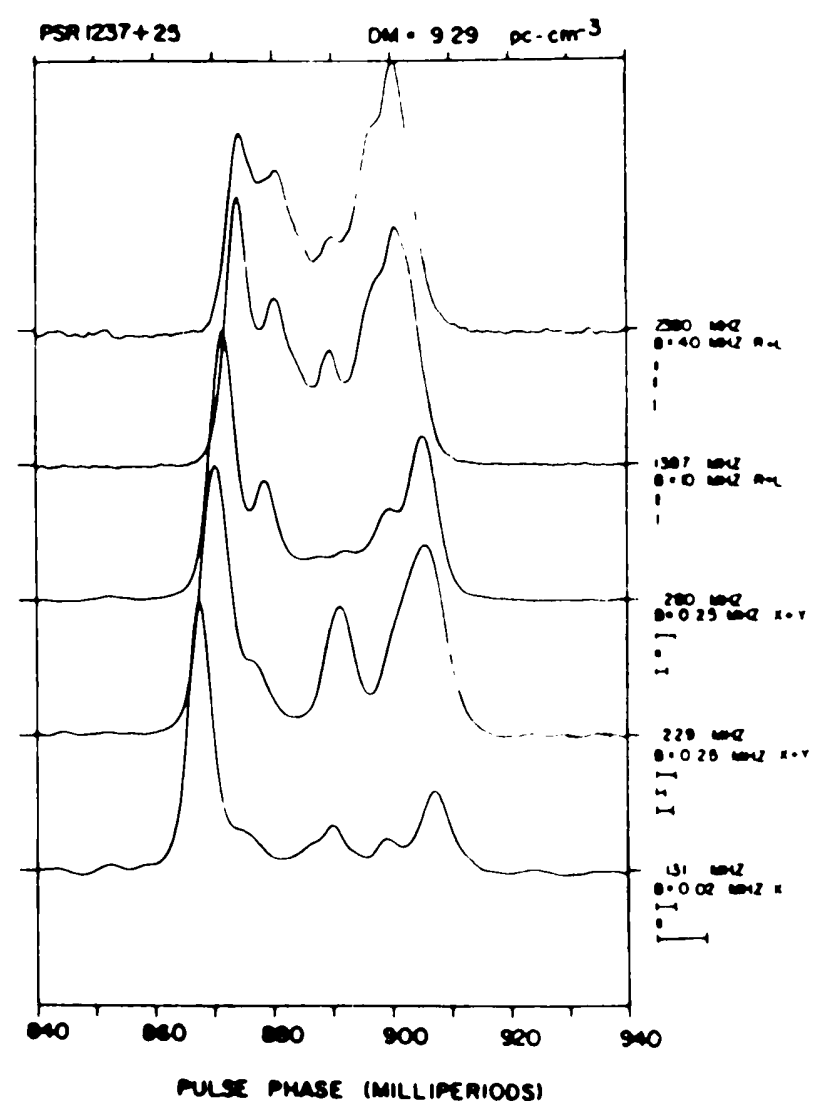

Figure 2 Composite total-power profiles for PSR $1237+25$ (from Hankins and Rickett 1986).

zone centrally. (PSR $1237+25$ 's polarization angle changes rather little, indicating an even more central traverse.)

- the sense of the circular polarization change is usually correlated with the sense of the linear polarization angle traverse as follows: lefthand circular to right-hand circular is correlated with a negative (clockwise) traverse, or right-hand circular to left-hand circular with a positive (counterclockwise) traverse,

- stationary subpulse modulation is associated with the conal components. Little or no actual subpulse motion or drift is observed.

- depolarization on the outside edges of the outer component pair caused by the "orthogonal" polarization modes

- many members show prominent pulse nulling

\section{Physical characteristics of the $M$ species}

Members of the five-component (M) species have a number of physical characteristics in common:
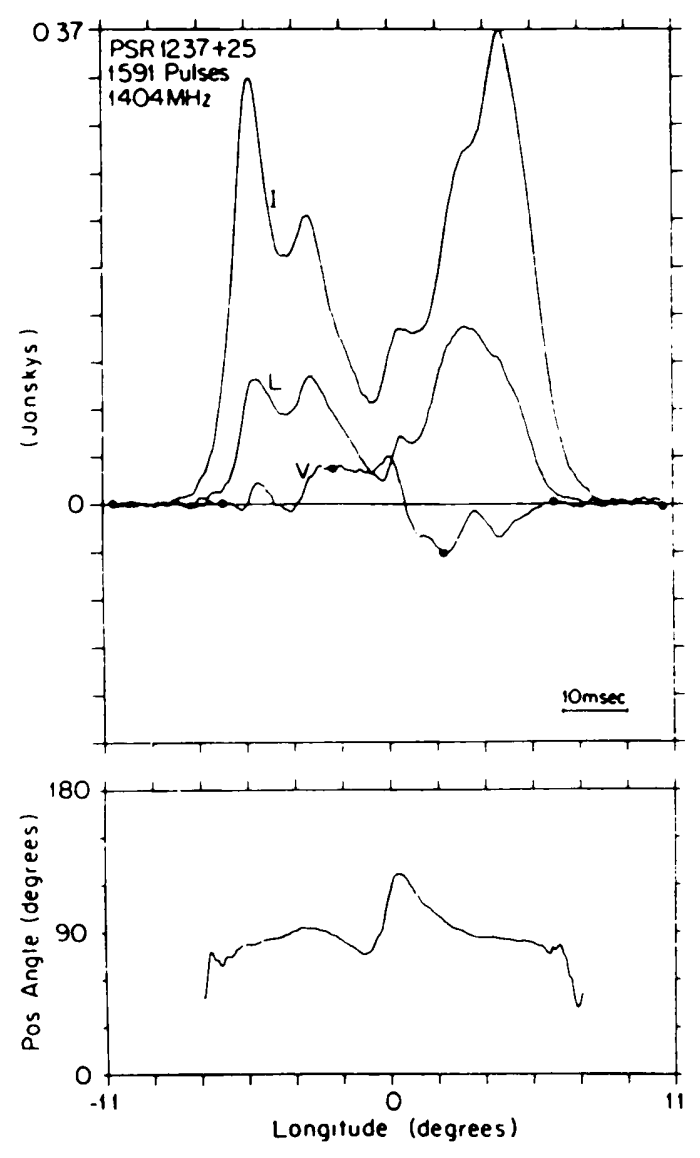

Figure 3 1400- $\mathrm{MHz}$ average polarization profile for PSR 1237+25 (from Stinebring et al. 1984)

- five-component pulsars are moderately old, but are not as old as the conal single $\left(\mathbf{S}_{\mathbf{d}}\right)$ and double (D) classes. They have a mean log age of 7.3 (as compared with about 7.7 for the $S_{d}$ and $D$ stars.;

- these stars have large galactic scale heights, more than $350 \mathrm{pc}$, compared with $210 \mathrm{pc}$ for the triple $(T)$ stars and $160 \mathrm{pc}$ for the $\mathbf{S}_{\mathrm{t}}$ pulsars.

- the five-component pulsars have fairly similar spindown characteristics. They fall in a narrow band of the P-P diagram-at moderately long periods and low spindown valuesparallel to the "death line". See figure 6.

- consequently, members of the $\mathbf{M}$ class have small to moderate values of the acceleration potential. This quantity, parameterized as $B_{12} / P^{2}$, generally takes values less than 2.5 and greater than 0.2 for these stars.

\section{Emission geometry calculations}

A study of core emission in pulsars with interpulses (Rankin 1990) results in the conclusion that core emission is produced throughout the polar-cap region - that is, near the surface of the star and over 

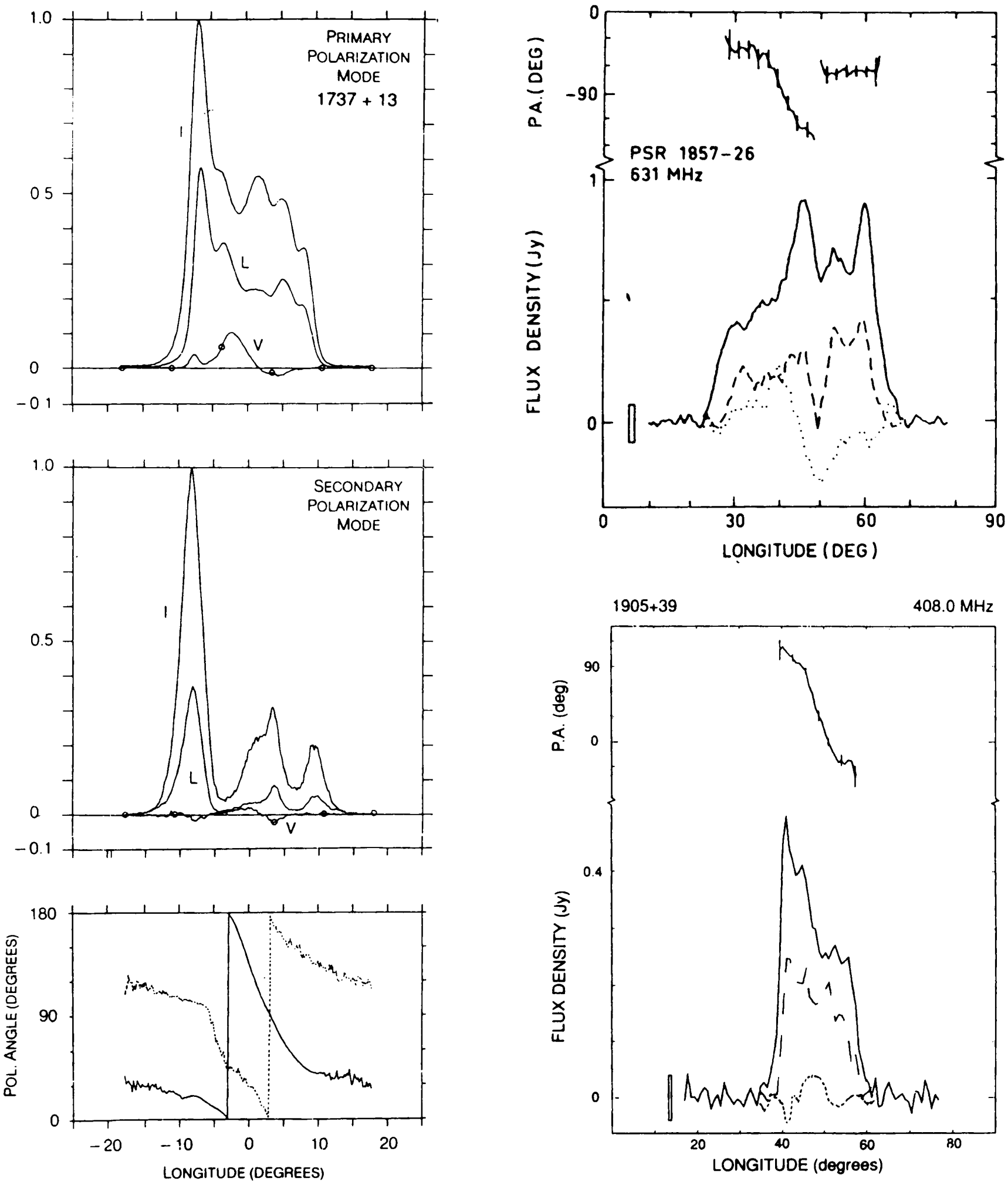

Figure 4 Three other examples of polarized average profiles of five-component (M) pulsars:

left PSR 1737+13 in both its "normal" and "abnormal" mode at $21 \mathrm{~cm}$ (from Rankin et al. 1988).

upper right PSR 1857-26 (from McCulloch et al. 1978).

lower right PSR 1905+39 (from Lyne and Manchester 1988).

the entire region defined by the "open" magnetic where $P$ is the rotation period in seconds and $\alpha$ the field lines.

The 1-GHz width of core components $W_{\text {core }}$ then appears to follow a simple scaling rule:

$$
W_{\text {core }}=2.45^{\circ} P^{-1 / 2} / \sin \alpha,
$$
angle between the rotation and magnetic axes. The angle $\alpha$ can then be determined for any pulsar with a core component.

When $\alpha$ is known, the radius of the conal emis(1) sion zone can be determined from the width of the 


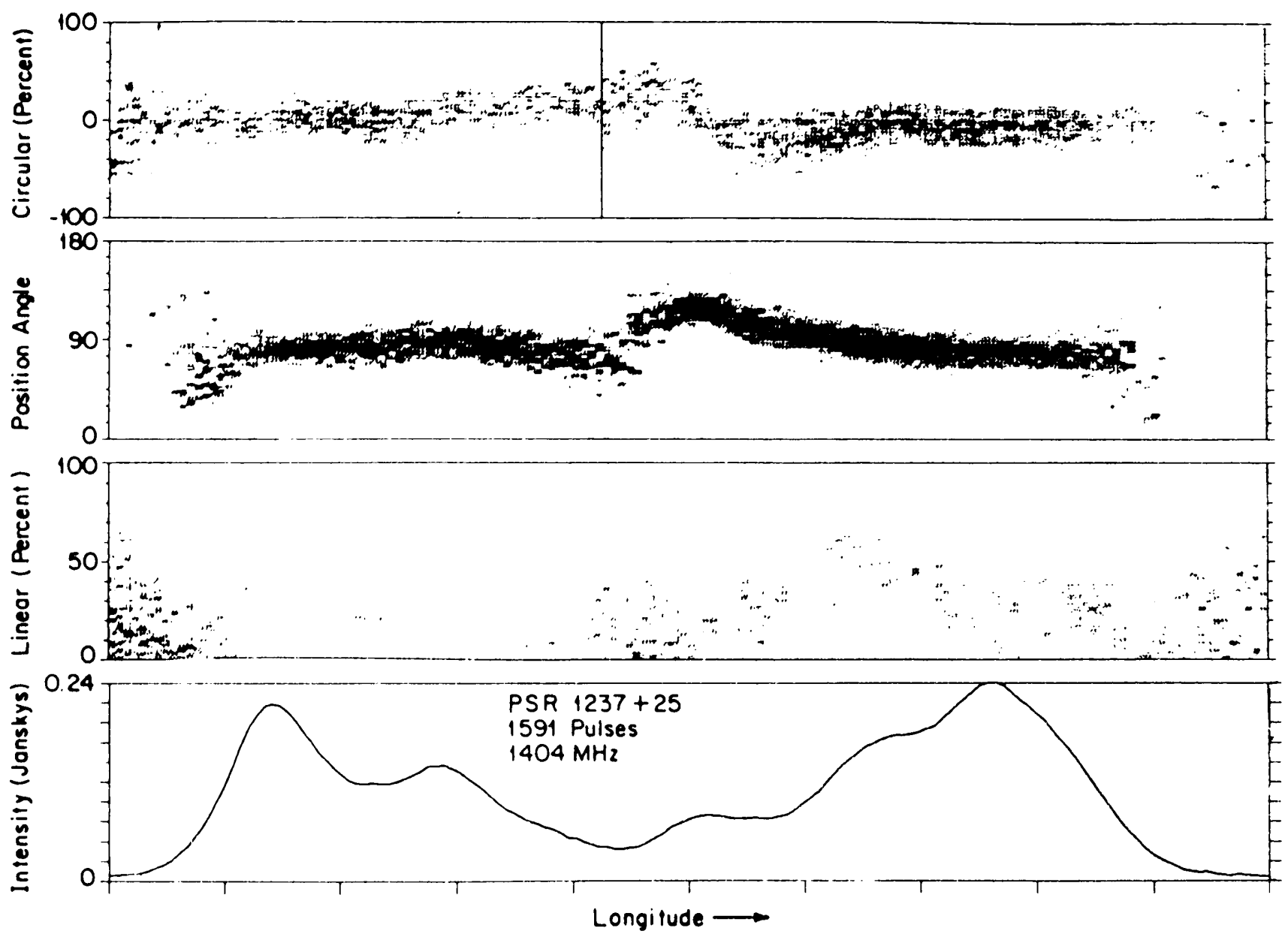

Figure 5 Histogram of the distribution of polarization for pulsar $1237+25$ (from Stinebring et al. 1984)

conal component pair and the impact angle between the sight line and the magnetic axis. This geometry is depicted in figure 7.

The full $1-\mathrm{GHz}$ width of the conal profile pair (to the outside half-power points), $\Delta \Psi$, is

$$
\Delta \Phi=\sin ^{-1}\left\{\frac{\left(\sin \left(\frac{\rho}{2}+\frac{\beta}{2}\right) \sin \left(\frac{\rho}{2}-\frac{\beta}{2}\right)\right)}{\sin \alpha \sin (\alpha+\beta)}\right\},
$$

where $\rho$ is the radius of the conal emission zone and $\beta$ the "impact angle" of the sight line with the magnetic axis. Solving for $\rho$, we have

$$
\cos \rho=\cos \beta-2 \sin \alpha \sin (\alpha+\beta) \sin ^{2}\left(\frac{\Delta \Phi}{4}\right) \text {. }
$$

The angle $\beta$ can then be calculated from $\alpha$ and the maximum rate of change of the linear polarization angle

$$
\left|\frac{\Delta \chi}{\Delta \phi}\right|_{\phi=0}=\frac{\sin \alpha}{\sin \beta},
$$

where $\chi$ is the polarization angle and $\phi$ is the longitude.

Given that the core emission is produced close to the $(R \simeq 10 \mathrm{~km})$ surface of the neutron star, the
1-GHz emission height of the conal radiation can be estimated via the following relation, which assumes a purely dipolar geometry in the emitting region

$$
h_{1 \mathrm{GHz}}=10 \mathrm{~km}\left(\frac{2 \rho}{W_{\text {core }}}\right)^{2}=10 \mathrm{~km}\left(\frac{2 \rho}{2.45^{\circ}} \frac{P^{-1 / 2}}{\sin \alpha}\right)^{2}
$$

\section{Geometry of emission}

The emission geometry of five-component (M) pulsars can be calculated with some confidence, owing to the presence of both a core component and pairs of conal components. The results of the calculations are shown in table 1. $\alpha$, the angle between the magnetic and rotational axes, can be determined from the $1-\mathrm{GHz}$ width of the core component using eq.(1) above. The derived values of $\alpha$ are found to fall between $3^{\circ}$ and $80^{\circ}$ with most around $30^{\circ}$. The $M$ stars are somewhat more aligned-that is, more have smaller values of $\alpha$-than either the triple (T) or core-single $\left(S_{t}\right)$ species.

The other values in the table are calculated using eqs.(3), (4) and.(5) above. As shown in figure 8 , the $1-\mathrm{GHz}$ angular radii of the inner and outer 


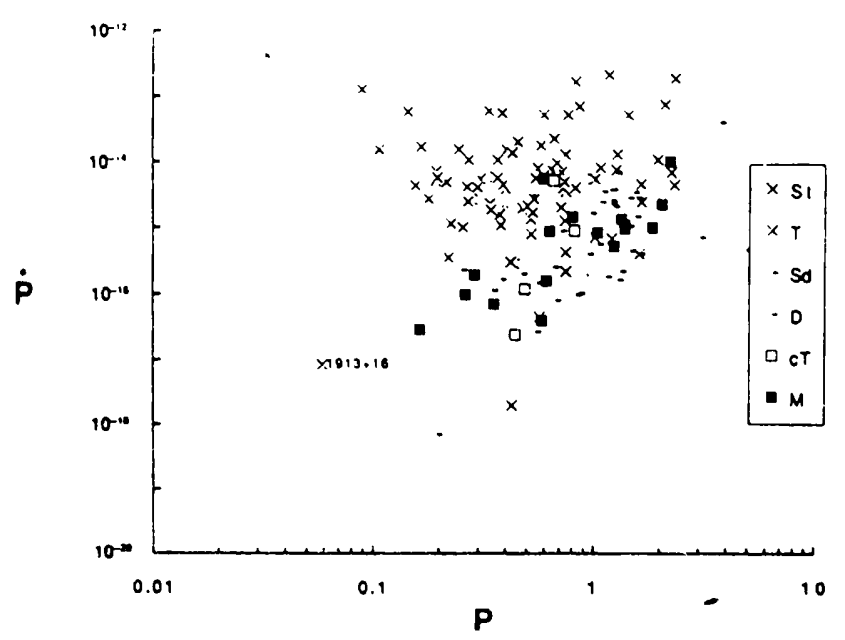

Figure $6 \quad P-\dot{P}$ diagram for some 160 pulsars. Note that the double-conal pulsars, the five-component $(\mathbf{M})$ and conal triple (cT), fall near the boundary between the core-single $\left(S_{t}\right)$ and triple $(T)$ pulsars and the conal single $\left(S_{d}\right)$ and double (D) stars.

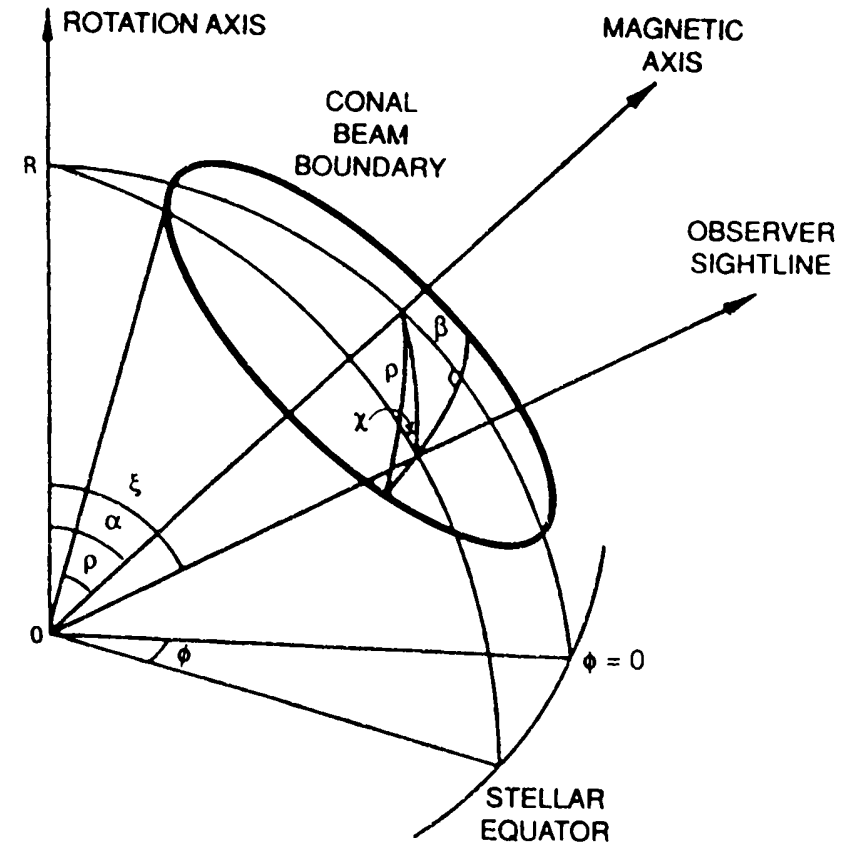

Figure 7 Diagram showing the geometry of the conal emission.

Table 1 The emission geometry of five-component (M) pulsars.

\begin{tabular}{|lcrrrrrrrrrrc|}
\hline Pulsar & Class & $\alpha$ & Sweep & $\beta$ & $\Delta \Psi_{1}$ & $\rho_{1}$ & $\beta / \rho_{1}$ & $\Delta \Psi_{2}$ & $\rho_{2}$ & $\beta / \rho_{2}$ & $h_{1}$ & $h_{2}$ \\
\hline $0402+61$ & $\mathrm{M} / \mathrm{T} ?$ & 83 & $+26 \dagger$ & 2.2 & $?$ & - & - & 14 & 7.3 & 0.30 & - & $(211)$ \\
$0523+11$ & $\mathrm{M} ?$ & 78 & $-9.5 \dagger$ & -5.9 & $?$ & - & - & 15.2 & 9.4 & -0.63 & - & $(211)$ \\
$0621-04$ & $\mathrm{M}$ & 32 & $\infty \dagger$ & 0 & $?$ & - & - & 22 & 5.9 & 0.00 & - & 238 \\
$0826-34 \mathrm{~m}$ & $\mathrm{M}$ & 3 & $+2.7 \dagger$ & 1.1 & $\sim 100 ?$ & 3.1 & 0.35 & 142 & 4.2 & 0.26 & $(120)$ & $(213)$ \\
$1039-19$ & $\mathrm{M}$ & 31 & $-18 \dagger$ & -1.7 & $\sim 13 ?$ & 3.7 & -0.45 & 17 & 4.6 & -0.36 & 126 & 197 \\
$1237+25$ & $\mathrm{M}$ & 53 & $\infty$ & 0.0 & 8.6 & 3.4 & 0.00 & 12.2 & 4.9 & 0.00 & 109 & 220 \\
$1451-68$ & $\mathrm{M} ?$ & 37 & -5.7 & -6.0 & 19.7 & 8.1 & -0.74 & 33.1 & 10.9 & -0.55 & 115 & 209 \\
$1737+13$ & $\mathrm{M}$ & 41 & -15 & -2.5 & $\sim 13$ & 4.8 & -0.52 & 19.4 & 6.6 & -0.38 & 124 & 235 \\
$1738-08$ & $\mathrm{M} ?$ & 24 & $+15 \dagger$ & 1.6 & $\sim 12$ & 3.0 & +0.53 & 17.2 & 3.9 & +0.40 & $(120)$ & $(211)$ \\
$1804-08$ & $\mathrm{M} / \mathrm{T}$ & 60 & -9 & -5.5 & $\sim 21$ & 10.4 & -0.53 & $\sim 30 ?$ & 13.7 & -0.40 & 118 & 206 \\
$1831-04$ & $\mathrm{M}$ & 12 & $-5 \dagger$ & -2.4 & $\sim 85 ?$ & 8.1 & -0.29 & 113 & 10.3 & -0.23 & 126 & 207 \\
$1857-26$ & $\mathrm{M}$ & 27 & -11 & -2.3 & $\sim 24 ?$ & 5.6 & -0.41 & 33 & 7.4 & -0.31 & 130 & 225 \\
$1905+39$ & $\mathrm{M}$ & 33 & $-15 \dagger$ & -2.1 & $\sim 12 ?$ & 3.8 & -0.55 & 16.4 & 4.9 & -0.43 & $(121)$ & $(195)$ \\
$1910+20$ & $\mathrm{M} / \mathrm{T} ?$ & 29 & +18 & 1.5 & 9.5 & 2.8 & +0.55 & 14 & 3.8 & +0.41 & 117 & 213 \\
$1919+21$ & $\mathrm{M} ?$ & 45 & \pm 11 & -3.7 & $\sim 4.0$ & 3.9 & -0.94 & 9.4 & 4.9 & -0.75 & 137 & 212 \\
$2003-08$ & $\mathrm{M}$ & 13 & +4 & 3.3 & $\sim 35 ?$ & 5.5 & +0.59 & 53 & 7.5 & +0.44 & $(119)$ & $(217)$ \\
$2028+22$ & $\mathrm{M} ?$ & 50 & \pm 8 & -5.5 & $?$ & - & - & 12.2 & 7.1 & -0.78 & - & 214 \\
$2210+29$ & $\mathrm{M}$ & 43 & $\infty \ddagger$ & 0.0 & 13 & 4.4 & 0.0 & 17 & 5.8 & 0.00 & 130 & $(223)$ \\
\hline
\end{tabular}

conal zones scale as $P^{-1 / 2}$ in the following manner

$$
\begin{aligned}
& \rho_{\text {inner }}=4.3^{\circ} P^{-1 / 2} \\
& \rho_{\text {outer }}=5.9^{\circ} P^{-1 / 2} .
\end{aligned}
$$

Finally, the 1-GHz emission heights of the inner and outer conal emission zones are

$$
\begin{aligned}
& h_{\text {inner }} \simeq 120 \mathrm{~km}, \text { or } \simeq 12 \text { stellar radii } \\
& h_{\text {outer }} \simeq 210 \mathrm{~km}, \text { or } \simeq 21 \text { stellar radii } .
\end{aligned}
$$

The core emission then comes from near the surface of the star, and the conal emission is radiated at a height of $100-200 \mathrm{~km}$ as indicated schematically in figure 9 . It is not clear whether the inner and outer conal zones are angular features in the emission pattern or a result of emission at different heights-as has been assumed here for purposes of illustration. 


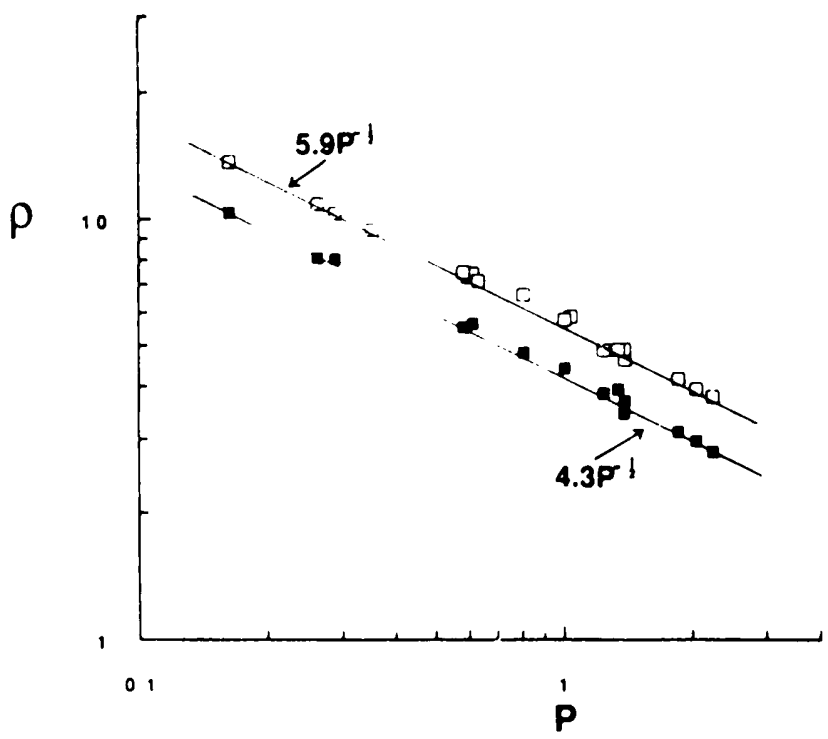

Figure 8 Conal radius $\rho$ as a function of period $P$ for the inner and outer conal zones of five-component (M) pulsars. The fitted curves $4.3^{\circ} P^{-1 / 2}$ and $5.9^{\circ} P^{-1 / 2}$ are indicated.

Neither is it as yet well understood why there are ever two conal zones of emission! There are several possibilities:

- the inner and outer zones represent emission by distinct currents. (Do the five-component profiles give us any hints regarding the path of the "return current"?)

- the inner conal zone represents conal emission that is "stimulated" by the core emission (and

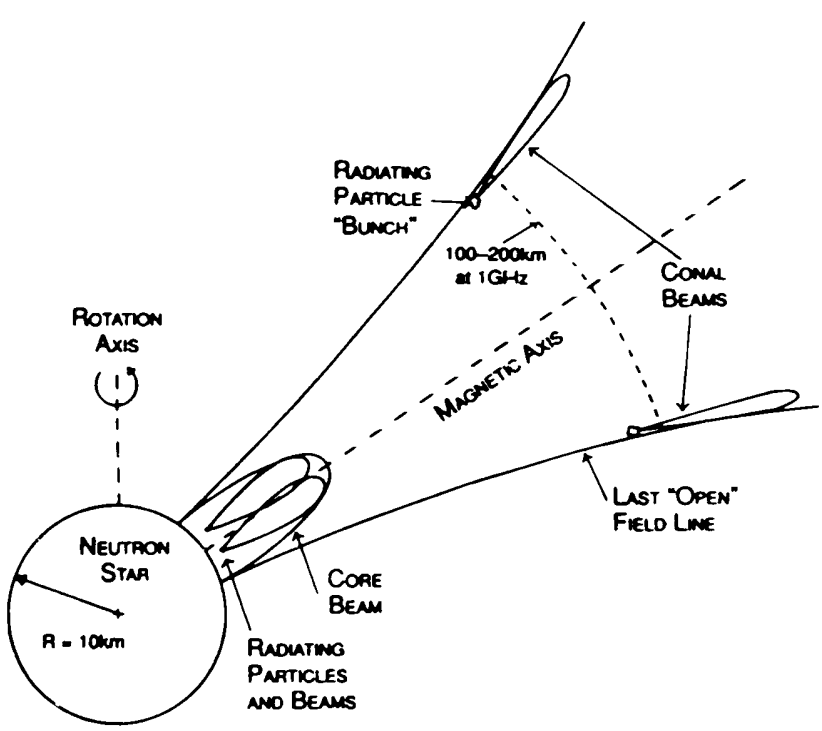

Figure 9 Cartoon of the core and conal emission geometry.

thus has much in common with the highfrequency conal components associated with core-single $\left(S_{t}\right)$ pulsars; whereas the outer conal zone has more in common with conal single $\left(\mathbf{S}_{d}\right)$ and double (D) profiles, in which core emission is apparently weak or absent.)

- the inner and outer conal zones represent distinct modes of propagation, either of radiofrequency or plasma waves, within the emission region. 